\title{
Shared and independent functions of aPKC $\lambda$ and Par3 in skin tumorigenesis
}

\author{
Susanne Vorhagen ${ }^{1,2,3} \cdot$ Dominik Kleefisch ${ }^{2,3}$ - Oana-Diana Persa ${ }^{1,2} \cdot$ Annika Graband $^{2,3} \cdot$ Alexandra Schwickert ${ }^{1,2,3}$. \\ Michael Saynisch ${ }^{2,3} \cdot$ Michael Leitges $^{4} \cdot$ Carien M. Niessen $^{1,2,3} \cdot$ Sandra Iden $^{2,3}$
}

Received: 15 August 2017 / Revised: 4 March 2018 / Accepted: 17 April 2018 / Published online: 23 May 2018

(c) The Author(s) 2018. This article is published with open access

\begin{abstract}
The polarity proteins Par3 and aPKC are key regulators of processes altered in cancer. Par3/aPKC are thought to dynamically interact with Par6 but increasing evidence suggests that aPKC and Par3 also exert complex-independent functions. Whereas aPKC $\lambda$ serves as tumor promotor, Par3 can either promote or suppress tumorigenesis. Here we asked whether and how Par3 and aPKC $\lambda$ genetically interact to control two-stage skin carcinogenesis. Epidermal loss of Par3, aPKC $\lambda$, or both, strongly reduced tumor multiplicity and increased latency but inhibited invasion to similar extents, indicating that Par3 and aPKC $\lambda$ function as a complex to promote tumorigenesis. Molecularly, Par3/aPKC $\lambda$ cooperate to promote Akt, ERK and NF- $\mathrm{KB}$ signaling during tumor initiation to sustain growth, whereas aPKC $\lambda$ dominates in promoting survival. In the inflammatory tumorigenesis phase Par3/aPKC $\lambda$ cooperate to drive Stat 3 activation and hyperproliferation. Unexpectedly, the reduced inflammatory signaling did not alter carcinogen-induced immune cell numbers but reduced IL-4 Receptor-positive stromal macrophage numbers in all mutant mice, suggesting that epidermal aPKC $\lambda$ and Par3 promote a tumor-permissive environment. Importantly, aPKC $\lambda$ also serves a distinct, carcinogen-independent role in controlling skin immune cell homeostasis. Collectively, our data demonstrates that Par3 and aPKC $\lambda$ cooperate to promote skin tumor initiation and progression, likely through sustaining growth, survival, and inflammatory signaling.
\end{abstract}

Shared first co-authors: Susanne Vorhagen, Dominik Kleefisch.

Shared senior authors: Carien M. Niessen, Sandra Iden.

Electronic supplementary material The online version of this article (https://doi.org/10.1038/s41388-018-0313-1) contains supplementary material, which is available to authorized users.

$\triangle$ Carien M. Niessen

carien.niessen@uni-koeln.de

$\triangle$ Sandra Iden

sandra.iden@uk-koeln.de

1 Department of Dermatology, University of Cologne, Köln, Germany

2 Cologne Excellence Cluster on Cellular Stress Responses in Aging-Associated Diseases (CECAD), Köln, Germany

3 Center for Molecular Medicine Cologne (CMMC), University of Cologne, Köln, Germany

4 Biotechnology Centre of Oslo, University of Oslo, 0316 Oslo, Norway

\section{Introduction}

One hallmark of carcinomas is the loss of cell polarity associated with altered epithelial organization and cell behavior. However, whether and how these alterations in epithelial organization drive malignant transformation and progression remains poorly understood. The cell polarity proteins Par3, Par6, and aPKC form the aPKC/Par complex and are key regulators of epithelial apico-basal polarity, migration, cell fate, and inflammation [1]. Most of the lossof-function data indicate that these proteins exert their function as part of this complex, albeit that in mammals a direct genetic comparison has not been done. Several findings also suggest that the complex is subject to highly dynamic regulation and that each of these proteins may serve complex-independent functions. For example, aPKC has other interaction partners that compete with either Par6 or Par3 binding, and Par3 dynamically interacts with aPKC/ Par6 in different functional settings [2, 3]. Recent evidence suggests that in breast cancer cells Par3 restricts aPKC function and downstream inflammatory signaling $[4,5]$. Finally, several observations indicate potentially 
independent roles for Par3 and aPKC, e.g., in DNA repair, inflammation, proliferation and epithelial structure [6-9]. Complicating matters further, for each protein several isoforms exist, encoded by different genes: $\mathrm{aPKC} \zeta$, aPKC $\lambda$, as well as Par3A and Par3B.

In human cancer, aPKC $\lambda$ is often overexpressed, whereas for Par3 both increased and decreased expression was observed [10-14], suggesting that these proteins have opposite actions also in human malignancies. Different mouse tumor models confirmed that aPKC $\lambda$ serves as a tumor promoter [15] whereas depending on the tumor settings Par3 indeed can serve both tumor-promoter and suppressor functions [4, 13, 15].

We previously identified Par3 as a promoter of 7,12Dimethylbenz(a)anthracene (DMBA)-induced epidermal skin cancer. Our in vitro data indicated that Par3 controls mutant Ras-driven proliferation through aPKC [13]. Par3 and $\mathrm{aPKC} \lambda$, the predominant aPKC isoform expressed in skin [16], are also major regulators of epidermal homeostasis. Loss of either protein resulted in hair follicle stem cell loss and increased differentiation, whereas these two proteins served opposite functions in regulating spindle orientation [16, 17], a mechanism implicated in epidermal stem/progenitor fate decisions [18, 19], suggesting both shared and distinct functions. Consequently, this tissue thus provides an excellent model system to directly address whether and how aPKC $\lambda$ and Par3 act in concert or independently in driving skin carcinogenesis. We therefore employed genetic loss-of-function mouse models and examined how loss of aPKC $\lambda$, Par3, or both, affect growth, apoptosis and inflammation at different stages of skin tumorigenesis.

\section{Results and discussion}

\section{Par3 and aPKC $\lambda$ cooperate in skin tumorigenesis}

To investigate whether aPKC $\lambda$ and Par3 cooperate or exert separate functions in tumor initiation and progression, single epidermal Par3 (Par3eKO) and $a P K C \lambda(a P K C \lambda \mathrm{eKO})$ as well as Par3/aPKC $\lambda$-double knockout mice (edKO) were generated using K14-Cre [13]-mediated recombination (Suppl.Fig. 1A), and subjected to DMBA/2-O-tetradecanoylphorbol-13-acetate(TPA)-mediated two-stage skin carcinogenesis regimen [20, 21]. DMBA induces mutations in a variety of genes, with predominant, activating mutations in Ras genes [22, 23]. In agreement, all tumors examined in this study were positive for the oncogenic Hras Q61L hot-spot mutation [20] (Suppl.Fig. 1B). TPA triggers hyperproliferation and inflammation, thereby promoting tumor outgrowth. Loss of aPKC $\lambda$ significantly reduced DMBA/TPA-induced papilloma multiplicity and delayed tumor formation (Fig. 1a, c, d), similar to loss of Par3 (Fig. $1 \mathrm{a}, \mathrm{c}, \mathrm{d})$ [13], identifying aPKC $\lambda$ as a tumor promoter in this non-melanoma skin cancer model. Importantly, combined loss of Par3 and aPKC $\lambda$ did not further reduce tumor burden or delay tumor incidence, providing genetic evidence that Par3 and aPKC $\lambda$ cooperate to promote skin tumorigenesis. Only at very late stages $a P K C \lambda \mathrm{eKO}$ mice showed a slightly reduced but significant difference in tumor numbers compared to Par3eKO and edKO mice (Fig. 1d), suggesting a tumor-suppressive role for Par3. Moreover, Par3 but not aPKC $\lambda$ loss promoted a significant increase in keratoacanthomas (Suppl.Fig. 1F). Together, these data confirm the previously reported dual role for Par3 in skin cancer [13]. Measurements of tumor sizes at 15 weeks post-DMBA revealed significantly smaller tumor diameters in Par3eKO, $a P K C \lambda \mathrm{eKO}$, and edKO mice compared to controls (Fig. 1b). Interestingly, although tumor burden, incidence and size were reduced, these tumors showed an increased invasive, albeit epithelial, phenotype (Suppl.Fig.1C-E). Importantly, immunohistochemical analysis revealed a significant upregulation of aPKC in human squamous cell carcinoma, suggesting that increased aPKC levels may also promote tumorigenesis in human skin cancer (Fig. 1e, f). Thus, mammalian aPKC $\lambda$ and Par3 act in concert to promote DMBA-induced skin carcinogenesis in vivo while serving an invasion-suppressive function at later stages of carcinogenesis.

\section{Increased apoptosis and sustained DNA damage upon DMBA treatment}

A critical step for DMBA-induced tumor initiation is whether mutant keratinocytes survive to give rise to skin tumors. To decipher if loss of Par3 and/or aPKC $\lambda$ affects cell survival, mice were subjected to a single topical dose of DMBA and analyzed $24 \mathrm{~h}$ later. Surprisingly, immunofluorescence analysis for phosphorylated histone $\mathrm{H} 2 \mathrm{Ax}$ $(\gamma \mathrm{H} 2 \mathrm{Ax})$, suggestive of sites of DNA damage, revealed a significant increase in $\gamma \mathrm{H} 2 \mathrm{Ax}$-positive cell nuclei in DMBA-treated $a P K C \lambda \mathrm{eKO}$ and edKO compared to control mice, with a less pronounced non-significant increase in Par3eKO mice (Fig. 2a, b). In agreement, apoptosis, as evidenced by cleaved Caspase3 (Fig. 2c, d), and DMBAinduced p53 (Suppl.Fig. 2C-E) was significantly increased in DMBA-treated $a P K C \lambda \mathrm{eKO}$ and edKO mice. These data might suggest that aPKC $\lambda$ plays a dominant role over Par3 in maintenance of cells harboring carcinogen-induced DNA lesions. Alternatively, as Par3 has previously been implicated in repair of $\gamma$-irradiation-induced DNA double-strand breaks [6], Par3 loss might elicit a similar effect but with different kinetics.

To investigate whether this increase in $\gamma \mathrm{H} 2 \mathrm{Ax}$ was accompanied by hyperproliferation, which may indicate 
a
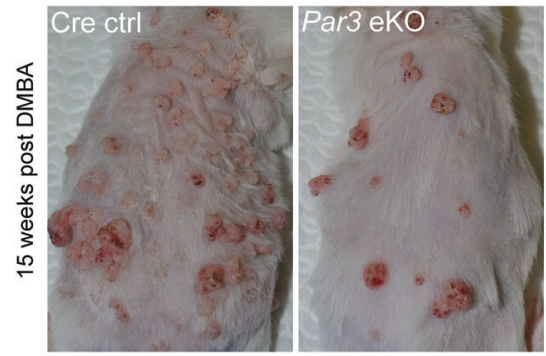

Tumor incidence
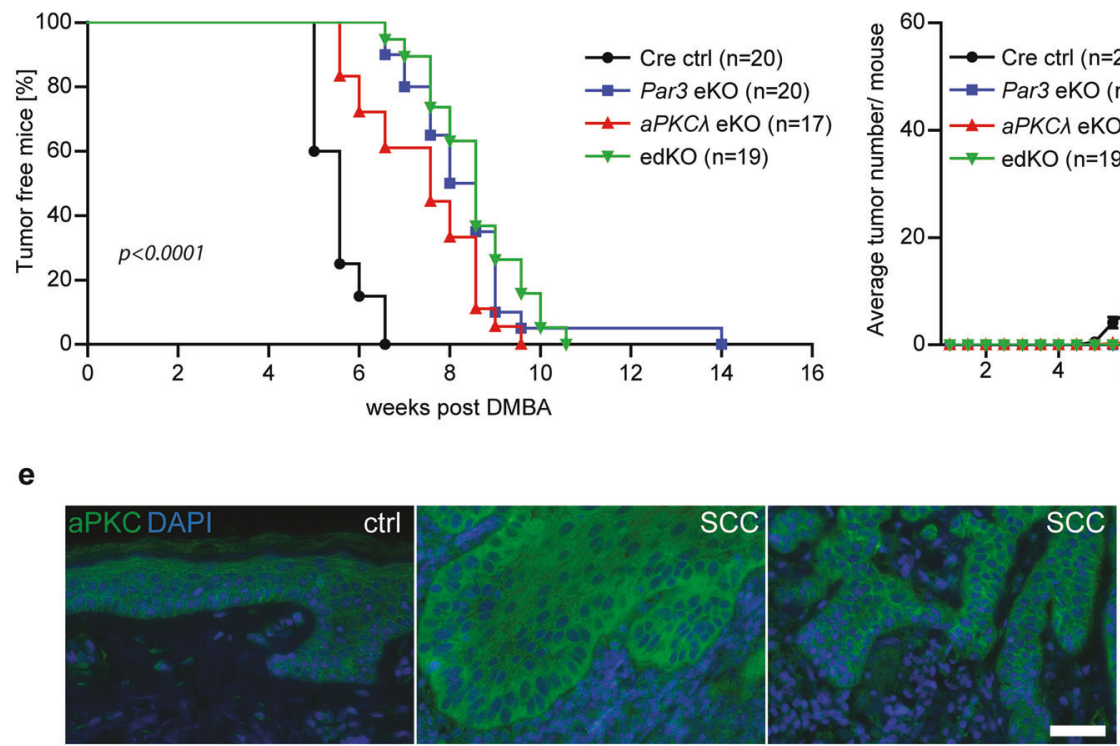

b Tumor sizes (15wks post DMBA)
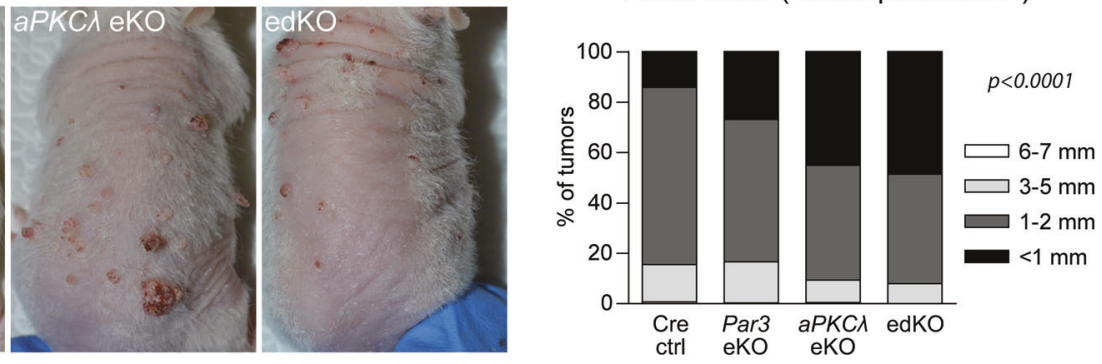

Tumor multiplicity

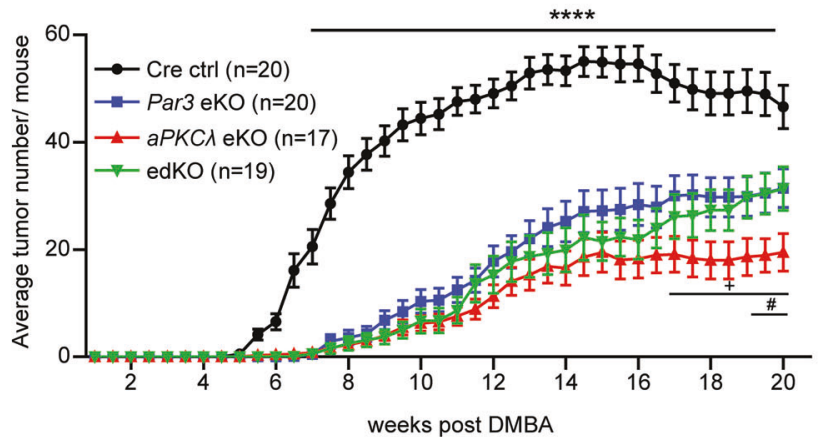

f
aPKC expression in human SCC

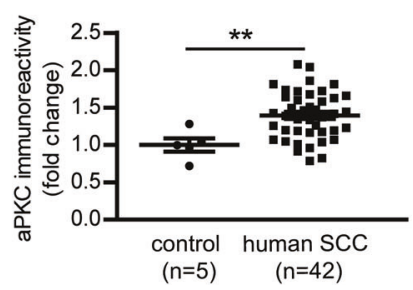

Fig. 1 Par3 and aPKC $\lambda$ cooperate in skin tumorigenesis. $\mathrm{Cre}^{+}$controls $\left(\mathrm{K} 14^{\mathrm{Cre} /+}\right)$, epidermis-specific Par3A (Par3eKO: K14 $\left.{ }^{\mathrm{Cre} /+} ; \mathrm{Par}^{\mathrm{f} / / \mathrm{f}}\right)$, $a P K C \lambda\left(a P K C \lambda \mathrm{eKO}: \mathrm{K} 14^{\mathrm{Cre} /+} ; a P K C \lambda^{\mathrm{f} / \mathrm{fl}}\right)$ and double-knockout mice (edKO: $\mathrm{K} 14^{\mathrm{Cre} /+} ; \operatorname{Par}^{\mathrm{fl} / \mathrm{fl}} ; a P K C \lambda^{\mathrm{fl} / \mathrm{fl}}$ ) were generated in a mixed FVBN/ C57BL/6N F2 background (K14-Cre [38]; Par3F [39]; $a P K C \lambda F$ [40]). Two independent two-stage skin carcinogenesis experiments were performed via long-term topical DMBA/TPA treatments as previously described [13]. 20 Cre ctrls, 20 Par3eKOs, 17 $a P K C \lambda$ eKOs, and 19 edKO mice were examined biweekly and tumor numbers and sizes were measured. Biometric analysis considering effect size, variation, type 1 and type 2 errors were performed to determine required group sizes that enable appropriate power. a Macroscopic tumor phenotypes of long-term DMBA/TPA-treated mice at 15 weeks after DMBA treatment. b Quantification of tumor sizes at 15 weeks post-DMBA treatment $(p<0.0001$; Chi square test with $4 \times 4$ contingency table). c Tumor incidence upon loss of Par3 and aPKC $\lambda$ in response to long-term DMBA/TPA treatment $(p<$ 0.0001; survival curves/ log-rank Mantel-Cox test; also $p<$ (control vs. each of the mutant lines) $<0.0001$ by "two-at-a-time" comparison followed by Bonferroni correction, and non-significant between individual mutant lines). d Tumor multiplicity upon loss of Par3 and aPKC $\lambda$ in response to long-term DMBA/TPA treatment (mean \pm SEM; 2 way-ANOVA/Tukey's post-hoc test; **** $p$ (control vs. each of the mutant lines $)<0.0001,+$ : $\mathrm{p}(a P K C \lambda \mathrm{eKO}$ vs. Par3eKO $)<0.05, \#: \mathrm{p}$ $(a P K C \lambda \mathrm{eKO}$ vs. Par3eKO and edKO) $<0.05$. Experiments were approved by and annually reported to the governmental authorities
("Landesamt für Natur, Umwelt und Verbraucherschutz NordrheinWestfalen," State Office North Rhine-Westphalia, Germany), and performed according to institutional guidelines and national animal welfare regulations. Sick, underdeveloped or by any other means atypical animals were excluded from the analyses. Mice of different genotypes were randomly co-housed. Males and females were used for experiments, with its ratio kept comparable between test and control groups. Mice of different litters derived from different parents were included in the analysis. The mouse IDs were unrelated to genotype, and a largely blinded analysis was performed e.g., for assessment of tumor initiation and growth in living mice, and subsequent dissection. e Immunofluorescence analysis as previously described [13] for atypical protein kinase C (aPKC) (sc-216, Santa Cruz Biotech.) on paraffin sections of a tissue microarray consisting of 42 samples for human squamous cell carcinoma (SCC) and 5 age-matched control skin samples (scale bar $=50 \mu \mathrm{m}$ ). The tissue microarray was constructed by collecting $2 \mathrm{~mm}$ tissue cores from paraffin-embedded samples and placed on host paraffin blocks using a manual tissue arrayer (MTA-1, AlphaMetrix Biotech). f Quantification of aPKC immunoreactivity in a tissue microarray of human SCC ( $n=5$ controls, $n=42$ human SCCs; mean $\pm \mathrm{SEM}$; ${ }^{*} p<0.01$; unpaired twosided $t$-test). Informed consent was obtained from all donors and patients involved in this study (BioMaSOTA form, University Hospital Cologne), and sampling of human specimen was approved by the ethic's committee of the Medical Faculty of the University of Cologne (file reference 12-163) 


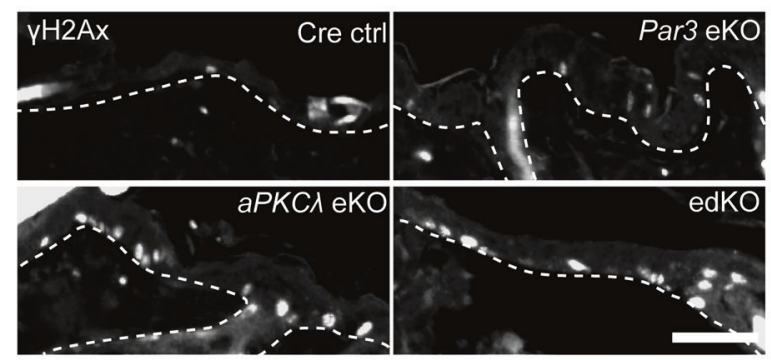

C
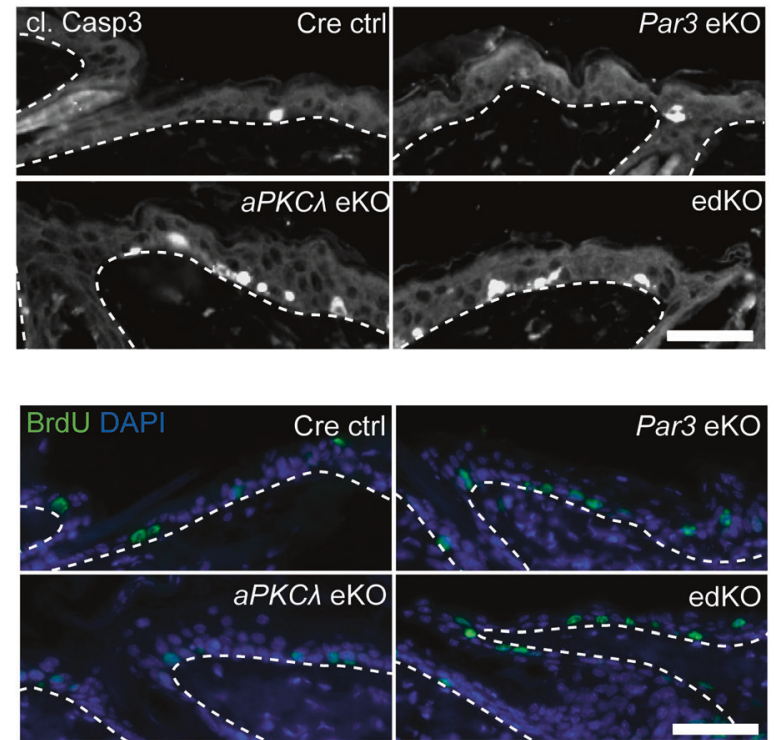
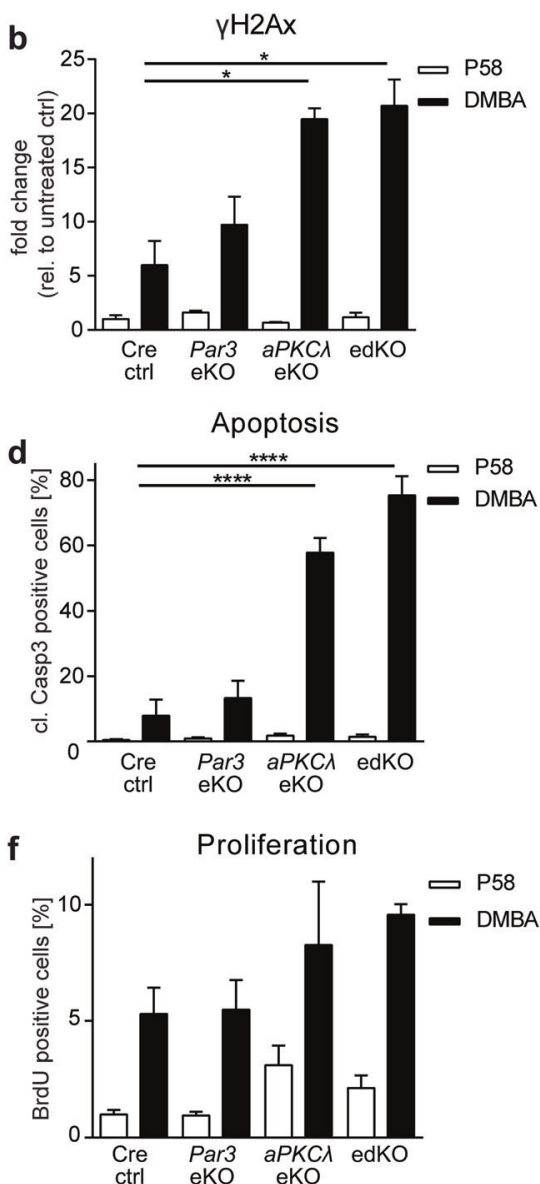

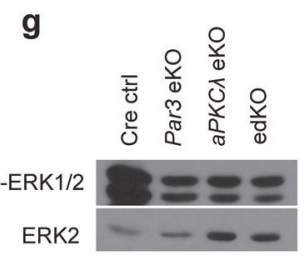

k

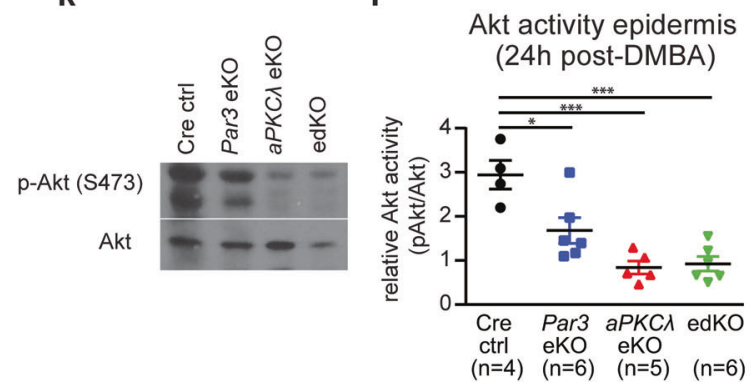

h ERK activity in epidermis (24h post-DMBA)

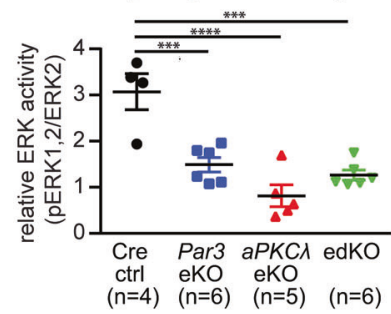

I

$(n=4) \quad(n=6) \quad(n=5) \quad(n=6)$ i ERK expression in epidermis j (24h post-DMBA)

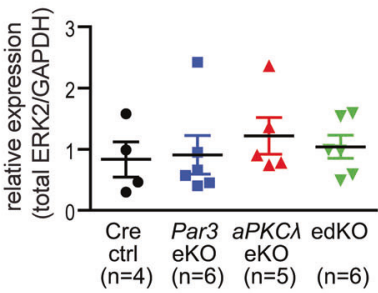

m

Akt expression in epidermis (24h post-DMBA)

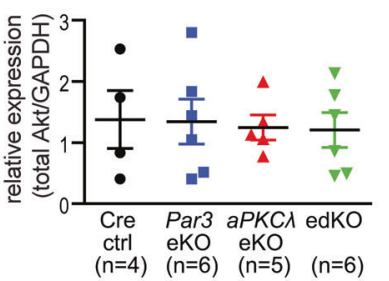

ERK activity in epidermis (P58 untreated)

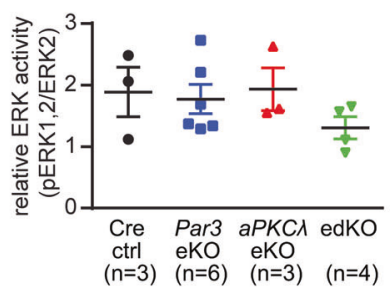

n

Akt activity in epidermis (P58 untreated)

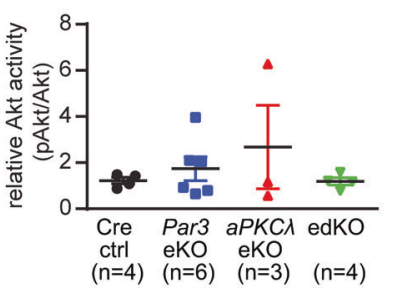

replicative stress, short-term BrdU incorporation or PCNA staining was assessed, which revealed no significant differences between any of the genotypes at $24 \mathrm{~h}$ post-DMBA
(Fig. 2e, f, Suppl.Fig. 2A, B). Together, these data indicate that epidermal loss of Par3 or aPKC $\lambda$ results in a differential sensitivity to both DNA damage and apoptosis, which, 
Fig. 2 Loss of Par3/aPKC $\lambda$ elicits increased apoptosis and sustained DNA damage upon DMBA treatment. For short-term DMBA treatments, mice were subjected to a single topical dose of DMBA at an age of 8 weeks ( $30 \mu \mathrm{g}$ in $200 \mu \mathrm{l}$ acetone, Sigma-Aldrich) and killed $24 \mathrm{~h}$ after treatment. a Immunofluorescence analysis for $\gamma \mathrm{H} 2 \mathrm{Ax}$ (Ab2893, Abcam) on paraffin sections of mice $24 \mathrm{~h}$ after DMBA treatment (scale bar $=50 \mu \mathrm{m}$ ) was performed as previously described [17], using $10 \%$ normal goat serum for blocking. b Quantification of $\gamma \mathrm{H} 2 \mathrm{Ax}$-positive cell nuclei in the epidermis of DMBA treated and untreated mice at P58 (fold change to untreated Cre ctrl $=1 ; n>8$ mice/ genotype; mean $+\mathrm{SEM} ; * p<0.05$; Kruskal-Wallis/Dunn's posthoc test). Tile-scans of entire tissue sections were generated (DM6000B; Leica Microsystems, Wetzlar, Germany) and $\gamma \mathrm{H} 2 \mathrm{Ax}-$ positive cell nuclei were manually counted, using DAPI as a counterstain. c Immunofluorescence analysis for cleaved Caspase3 (cl. Casp3) (\#9664, Cell signaling) on paraffin sections of mice $24 \mathrm{~h}$ after DMBA treatment (scale bar $=50 \mu \mathrm{m}$ ) as previously described $(2 \mathrm{~A})$. $\mathbf{d}$ Quantification of cl. Casp3-positive cell nuclei in the epidermis of DMBA treated and untreated mice at P58 as described in 2B (\% of DAPI-positive cell nuclei; $n>9$ mice/genotype; mean $+\mathrm{SEM} ; * * * p$ $<0.0001$; one-way ANOVA/Dunnett's post-hoc test). e Immunofluorescence analysis as described in (a) for BrdU (\#347580, BD Bioscience) on paraffin sections of mice $24 \mathrm{~h}$ after DMBA treatment (scale bar $=50 \mu \mathrm{m}$ ). Nuclei were counterstained with DAPI. Mice were injected intraperitoneally using $25 \mu \mathrm{g}$ BrdU (Calbiochem) per $\mathrm{kg}$ bodyweight $1 \mathrm{~h}$ prior to killing. $\mathbf{f}$ Quantification of BrdU-positive cells in the basal layer of DMBA treated and untreated mice at P58 as described in $2 \mathrm{~B}$ ( $\%$ of DAPI positive cell nuclei; $n>4 /$ genotype; mean + SEM, one-way ANOVA/Dunnett's post-hoc test). g Example and $\mathbf{h}$ quantification of western blot analysis for ERK activity ( $\mathrm{p}$ ERK1/2/ ERK2) (Cell Signaling Techn., \#9101 and BD Biosciences, \#610104) on epidermal lysates of mice $24 \mathrm{~h}$ after DMBA treatment. Epidermal-dermal separation of back-skin, measurement of protein concentration, SDS-PAGE, wet transfer, and Western Blot were performed as previously described [17]. Densitometry of protein bands was performed using ImageJ (Version $1.50 \mathrm{~g}$; National Institutes of Health, USA) on scanned western blots. Phosphorylated protein was normalized to total protein levels and total protein to GAPDH (Millipore, \#MAB374), used as loading control (mean $\pm \mathrm{SEM} ; * * * p<$ $0.001 ; * * * * p<0.0001$; one-way ANOVA/Tukey's post-hoc test). i Quantification of western blot analysis for ERK expression (ERK2/ GAPDH) (BD Biosciences, \#610104, Millipore MAB374) on epidermal lysates of mice $24 \mathrm{~h}$ after DMBA treatment (mean \pm SEM; oneway ANOVA/Tukey's post-hoc test). j Quantification of western blot analysis for ERK activity (p-ERK1/2/ ERK2) in untreated P58 mice as described in $\mathrm{h}$ (mean $\pm \mathrm{SEM}$; one-way ANOVA/Tukey's post-hoc test). $\mathbf{k}$ Example and $\mathbf{l}$ quantification of western blot analysis for Akt activity (pAkt (S473)/ Akt) (Cell Signaling Techn., \#4060, \#9272) on epidermal lysates of mice $24 \mathrm{~h}$ after DMBA treatment. GAPDH was used as loading control (mean \pm SEM; $* * * p<0.001$; $* p<0.05$; oneway ANOVA/Tukey's post-hoc test). $\mathbf{m}$ Quantification of western blot analysis for Akt expression (Akt/GAPDH) (Cell Signaling Techn., \#9272, Millipore MAB374) on epidermal lysates of mice $24 \mathrm{~h}$ after DMBA treatment (mean \pm SEM; one-way ANOVA/Tukey's post-hoc test). n Quantification of western blot analysis for Akt activity (p-Akt/ Akt) in untreated P58 mice as described in $\mathbf{l}$ (mean \pm SEM; one-way ANOVA/Tukey's post-hoc test). Technical assessment in terms of staining specificity, transfer efficiency for immunoblots and others was performed each time, whereby positive (where available) and negative controls were used for evaluation. For quantification of different properties (apoptosis, oncogenic signaling, BrdU incorporation, protein expression) blinding was implemented at the level of micrographs that were captured for further analyses. Additionally, automated and unbiased approached, e.g., using CellProfiler and ImageJ software, was used to obtain most objective results. See Suppl. Table 1 for antibody dilutions however, cannot explain the similar tumor incidence and multiplicity seen in Par3eKO, $a P K C \lambda \mathrm{eKO}$, and edKO mice.

\section{Par3/aPKC promote growth and survival signaling following DMBA exposure}

We next asked how loss of aPKC $\lambda$ and/or Par3 counteract tumor initiation and promotion, and investigated different signaling cascades important for two-stage skin carcinogenesis [24]. Western Blot analysis revealed a similar, significant reduction in ERK1/2 activity, but not levels, in DMBA-treated Par3eKO, aPKC $\mathrm{e} \mathrm{KO}$, and edKO mice compared to controls (Fig. 2g-i), which was not observed in non-treated epidermis (Fig. 2j, Suppl.Fig. 2F). Thus, in line with our previous in vitro results [13], aPKC $\lambda$ and Par3 function in concert to stimulate ERK in vivo under carcinogenesis-inducing conditions.

Akt activation promotes keratinocyte survival in DMBAinduced skin carcinogenesis [25]. Interestingly, loss of Par3, aPKC $\lambda$, or both resulted in significantly reduced in vivo Akt activity, but not levels (Fig. 2k-m), which was most prominent in $a P K C \lambda \mathrm{eKO}$ or edKO mice, potentially explaining the increased apoptosis in these mice (Fig. 2d). No change in Akt activity and levels was observed in untreated mice irrespective of genotype (Fig. 2n, Suppl.Fig. 2I). Together, these results indicate that the aPKC $\lambda / P a r 3$ complex is essential to induce early growth and survival signaling in response to DMBA.

\section{Reduced NF-kB and Stat3 activation upon Par3/ aPKCX loss}

One hallmark of non-melanoma skin cancer is an inflammatory tumor-promoting micro-environment, faithfully reproduced by the DMBA/TPA skin cancer protocol [21, 22]. Potent regulators of inflammatory signals are NF- $\mathrm{kB}$ and Stat3 $[26,27]$. Loss of the NF- $\mathrm{KB}$ subunit p65 impaired immune cell recruitment during Ras-mediated skin carcinogenesis [28]. Both aPKC $\lambda$ and Par3 have been implicated in NF- $\mathrm{KB}$ signaling even though conflicting data exist whether they exert inhibitory or activating signals controlling NF- $\mathrm{KB}$ and immune responses [29, 30]. Interestingly, DMBA-treated Par3eKO, aPKC $\mathrm{eKO}$, and edKO mice displayed an increased number of nuclear pS468-p65positive cells, indicative of reduced NF-KB signaling [31], as well as reduced total p65 protein levels, albeit only statistically significant for $a P K C \lambda \mathrm{eKO}$ and edKO mice, with no significant changes in non-treated skin (Fig. 3a, b, Suppl. Fig. 3A-C).

NF- $\mathrm{KB}$ cooperates with Stat3 signaling in different cancers [32]. Constitutive Stat3 activation fosters skin tumor progression [33], whereas its loss impairs skin 
a p-p65 (S468) in epidermis

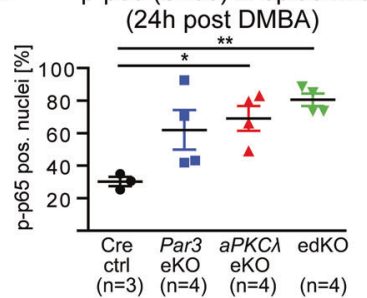

b p65 expression in epidermis

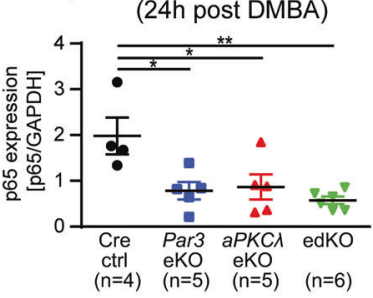

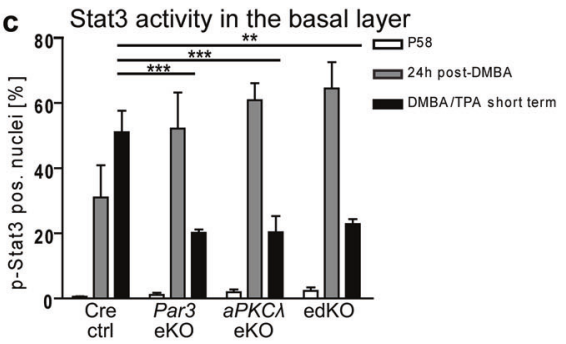

d CD45 dermis (untreated) i CD45 dermis (DMBA/TPA)

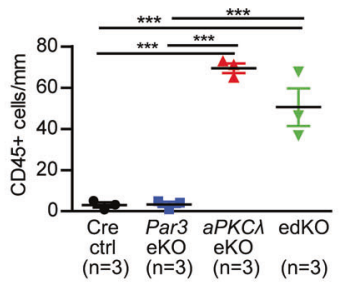

e CD45 epidermis (untreated)

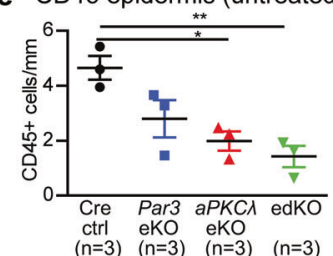

$(n=3) \quad(n=3) \quad(n=3) \quad(n=3)$

f $\mathrm{CD} 3$ dermis (untreated)

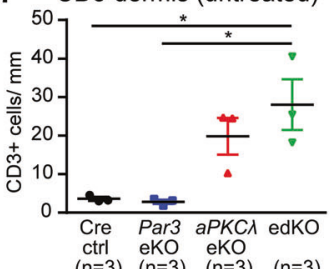

$(n=3) \quad(n=3) \quad(n=3) \quad(n=3)$

g CD3 epidermis (untreated)

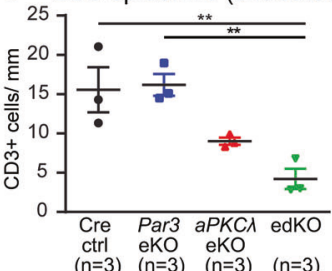

h

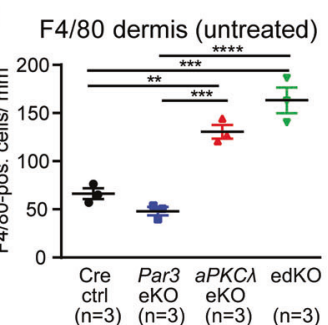

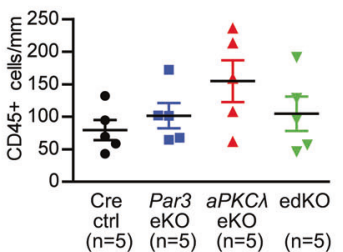

j CD45 epidermis (DMBA/TPA)

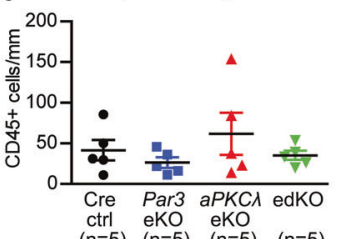

$(n=5) \quad(n=5) \quad(n=5) \quad(n=5)$

k CD3 dermis (DMBA/TPA)

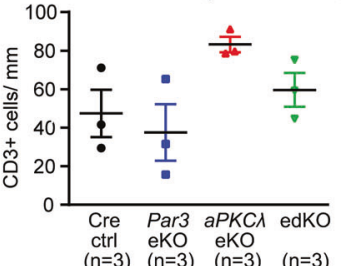

$(n=3) \quad(n=3) \quad(n=3) \quad(n=3)$
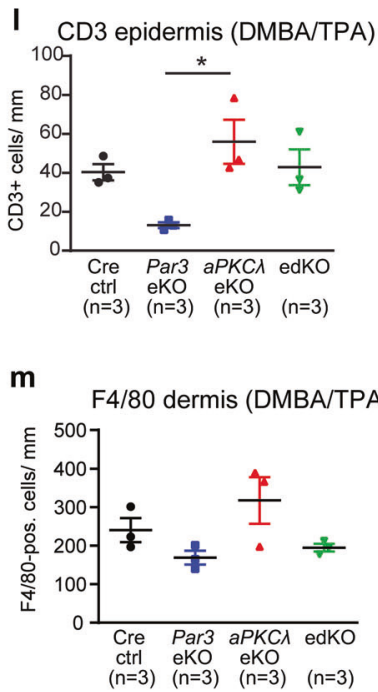

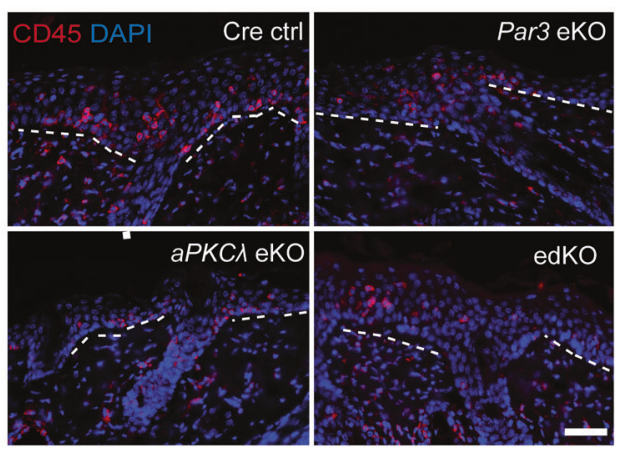

$\circ$

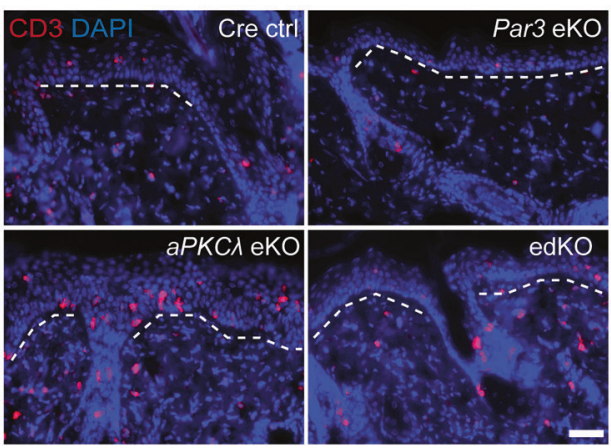

p

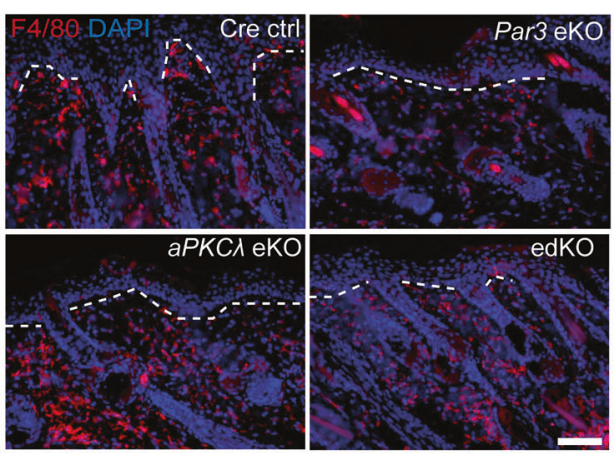


Fig. 3 Par3 and aPKC $\lambda$ control carcinogen-induced inflammatory signaling but not early immune cell recruitment. a Quantification of immunofluorescence analysis as previously described (2B) for p-p65 (S468) (Cell Signaling Techn. \#3039) on paraffin sections of mice $24 \mathrm{~h}$ after DMBA treatment ( $\%$ of DAPI positive cell nuclei; mean \pm SEM; ${ }^{*} p<0.05, * * p<0.01$; one-way ANOVA/Tukey's post-hoc test). b Quantification of western blot analysis for total p65 protein in epidermal lysates of mice $24 \mathrm{~h}$ after DMBA treatment (mean \pm SEM; $* p$ $<0.05$, ** $p<0.01$; one-way ANOVA/Tukey's post-hoc test). Sample preparation and western blot analysis was performed as previously described [17]. c Quantification of immunofluorescence analysis as previously described (2B) for p-STAT3 (Y705) (Cell Signaling Techn. \#9145) on paraffin sections of short-term DMBA/TPA treated mice (\% of DAPI positive cell nuclei; mean + SEM; $* * p<0.01, * * * p<0.001$; P58: $n($ Cre ctrl $)=5, n($ Par3eKO $)=4, n(a P K C \lambda \mathrm{eKO})=3, n(\mathrm{edKO})$ $=5 ; 24 \mathrm{~h}$ post-DMBA: $n(C r e \operatorname{ctrl})=3, n(\operatorname{Par} 3 \mathrm{eKO})=4, n(a P K-$ $C \lambda \mathrm{eKO})=4, n(\mathrm{edKO})=3$; short-term DMBA/TPA: $n($ Cre ctrl $)=5, n$ $($ Par3eKO $)=5, n($ aPKC $\lambda \mathrm{eKO})=4, n(\mathrm{edKO})=4$; one-way ANOVA/ Tukey's post-hoc). For short-term DMBA/TPA treatments, at P58 mice were treated with a single dose of DMBA $(40 \mu \mathrm{g}$ in $200 \mu \mathrm{l}$ acetone, Sigma) and three applications of TPA (200 $\mu$ l of $10^{-4} \mathrm{M}$ TPA/ acetone) at P65, 68 and 72, and killed 2 days after the last TPA treatment. d Quantification of CD45-positive cells $/ \mathrm{mm}$ dermis of untreated mice as described in $2 \mathrm{~B}$ (mean $\pm \mathrm{SEM}$; $* * * p<0.001$; oneway ANOVA/Tukey's post-hoc test). e Quantification of CD45positive cells/ mm epidermis of untreated mice (mean \pm SEM; $* p<$ $0.05, * * p<0.01$; one-way ANOVA/Tukey's post-hoc test). f Quantification of CD3-positive cells/ mm dermis of untreated mice (mean \pm SEM; one-way ANOVA; Tukey's post-hoc test, $* p<0.05)$. g Quantification of CD3-positive cells/ $\mathrm{mm}$ epidermis of untreated mice (mean $\pm \mathrm{SEM}$; one-way ANOVA/Tukey's post-hoc test, $* p<0.05$ ). h Quantification of F4/80-positive cells/ $\mathrm{mm}$ dermis of untreated mice (mean \pm SEM; $\quad * * p<0.01 ; \quad * * * p<0.001 ; \quad * * * p<0.001 ;$ one-way ANOVA; Tukey's post-hoc test). i Quantification of CD45-positive cells/mm dermis of short-term DMBA/TPA treated mice (mean \pm SEM; one-way ANOVA/Tukey's post-hoc test). $\mathbf{j}$ Quantification of CD45-positive cells/ mm epidermis of short-term DMBA/TPA treated mice (mean $\pm \mathrm{SEM}$; one-way ANOVA/Tukey's post-hoc test). $\mathbf{k}$ Quantification of CD3-positive cells/mm dermis of short term DMBA/ TPA treated mice (mean \pm SEM, one-way ANOVA/Tukey's post-hoc test). I Quantification of CD3-positive cells $/ \mathrm{mm}$ epidermis of shortterm DMBA/TPA treated mice (mean $\pm \mathrm{SEM}, * p<0.05$, one-way ANOVA/Tukey's post-hoc test). m Quantification of F4/80-positive cells/mm dermis of short term DMBA/TPA-treated mice (mean \pm SEM, one-way ANOVA/Tukey's post-hoc test). n Immunofluorescence analysis for CD45 (12-0451-82, Invitrogen) on paraffin sections of short-term DMBA/TPA-treated mice (scale bar $=50 \mu \mathrm{m})$. Immunofluorescence analysis was performed as described in $1 \mathrm{E}$. Nuclei were counterstained with DAPI. o Immunofluorescence analysis for CD3 (AM11102PU-S, Acris) on paraffin sections of shortterm DMBA/TPA-treated mice (scale bar: $50 \mu \mathrm{m}$ ). Immunofluorescence analysis was performed as previously described including additional antigen retrieval prior to blocking via incubation in $3 \%$ $\mathrm{H}_{2} \mathrm{O}_{2} / 0.5 \% \mathrm{KOH}$ (Sigma-Aldrich) for $30 \mathrm{~min}$ at $37^{\circ} \mathrm{C}$. Nuclei were counterstained with DAPI. p Immunofluorescence analysis for F4/80 (MCA497PET, ABD serotec) as previously described (3D) on paraffin sections of short-term DMBA/TPA-treated mice (scale bar $=100 \mu \mathrm{m})$. Nuclei were counterstained with DAPI. See Suppl. Table 1 for antibody dilutions

tumorigenesis [34]. Intriguingly, while Stat3 activity was similar in untreated and $24 \mathrm{~h}$ DMBA-treated epidermis (Fig. $3 c$ ), it was significantly reduced in all polarity mutant mice subjected to DMBA combined with three subsequent doses of TPA ("short-term DMBA/TPA") (Fig. 3c, Suppl.Fig. 3D). Thus, aPKC $\lambda$ and Par3 act in concert in skin carcinogenesis to promote cancer-associated $\mathrm{NF}-\mathrm{kB}$ and Stat3 signals. Our results agree with observations that depletion of either aPKC $\lambda$ in prostate cancer cells [35], or of Par3 in lung squamous cell carcinoma cells and ovarian cancer cells impaired Stat 3 activity [10, 36], but contrast findings in breast cancer cells, in which Par3 inhibits aPKC and, as consequence, NF-KB/Stat 3 activation $[4,5]$. Thus, different tumor entities elicit either cooperative or antagonizing Stat 3 responses of aPKC $\lambda$ and Par3. Together, the data strongly suggest that Par3 and aPKC $\lambda$ not only promote tumor initiation but also TPA-mediated inflammatory responses.

\section{Distinct role of aPKC $\lambda$ in controlling skin immune homeostasis, whereas overall DMBA/TPA-induced immune cell infiltration is Par3/aPKC $\lambda$-independent}

To address whether loss of Par3, aPKC $\lambda$, or both would affect immune cell homeostasis already in untreated skin, we performed immunohistochemical staining for CD45 (pan-leukocyte marker), CD3 (T-cells) and F4/80 (myeloid cells, mostly macrophages). Interestingly, immunofluorescence analysis revealed a strongly increased number of $\mathrm{CD} 45^{+}$cells (Fig. 3d, Suppl.Fig. 3F), $\mathrm{CD}^{+}$cells (Fig. 3f) and $\mathrm{F} 4 / 80^{+}$cells (Fig. $3 \mathrm{~h}$ ) in the dermis of untreated P58 aPKC $\mathrm{eKO}$ and edKO mice but not in control or Par3eKO mice. In contrast, in the epidermis immune cell numbers and, more specifically, $\mathrm{CD}^{+} \mathrm{T}$-cell numbers, were reduced upon loss of aPKC $\lambda$ or both aPKC $\lambda /$ Par3 (Fig. 3e, g). These data indicate a Par3-independent role for aPKC $\lambda$ in immune cell recruitment at homeostatic conditions. Short-term DMBA/TPA treatment resulted in similar numbers of dermal and largely similar epidermal immune cells $\left(\mathrm{CD} 45^{+}\right.$, Fig. 3i, j, n), and more specifically T-cells (Fig. 3k, 1, o) or macrophages (Fig. 3m, p) in all genotypes. Moreover, staining for markers that suggest tumor-promoting (IL4R) or tumor-suppressive (iNOS) macrophage identity [37] did not reveal any consistent changes either (data not shown). Thus, despite reduced epidermal inflammatory signals, and in contrast to epidermal $p 65 \mathrm{eKO}$ mice [28], the reduced tumor burden in Par3/aPKC $\lambda$-single or double-mutant mice cannot directly be attributed to an early reduction in infiltrating immune cells.

\section{Par3 and aPKC $\lambda$ control sustained DMBA/TPA- induced proliferation and survival}

We next asked whether aPKC $\lambda$ and Par3 cooperate in the outgrowth of Hras-mutated keratinocytes. As expected, short-term DMBA/TPA treatment strongly induced 

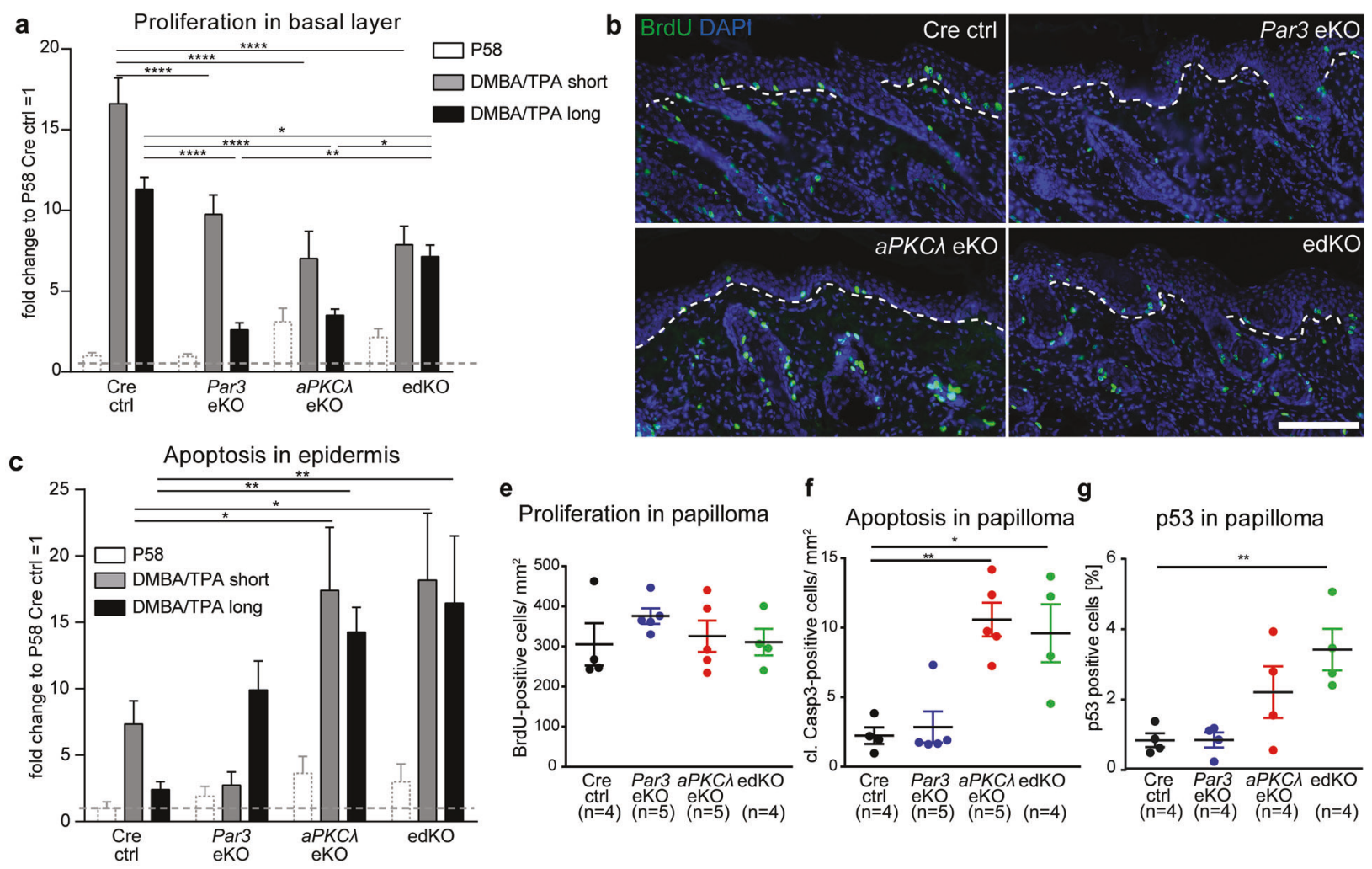

\section{d}

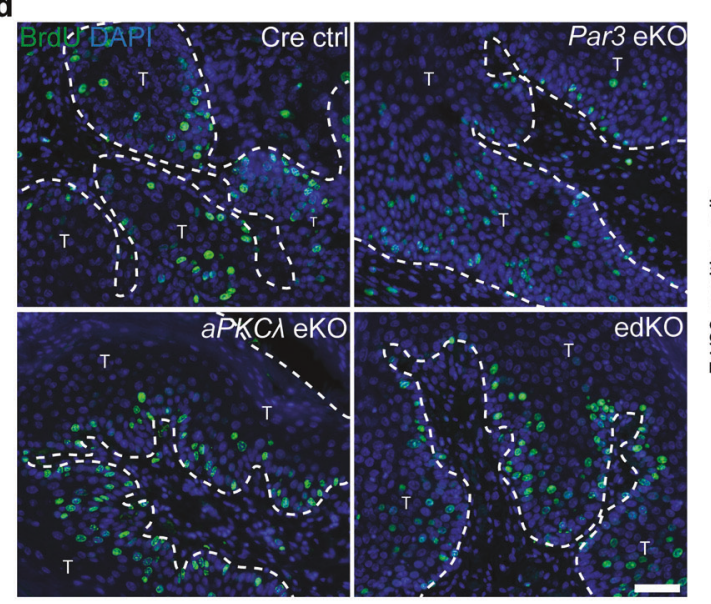

h

i
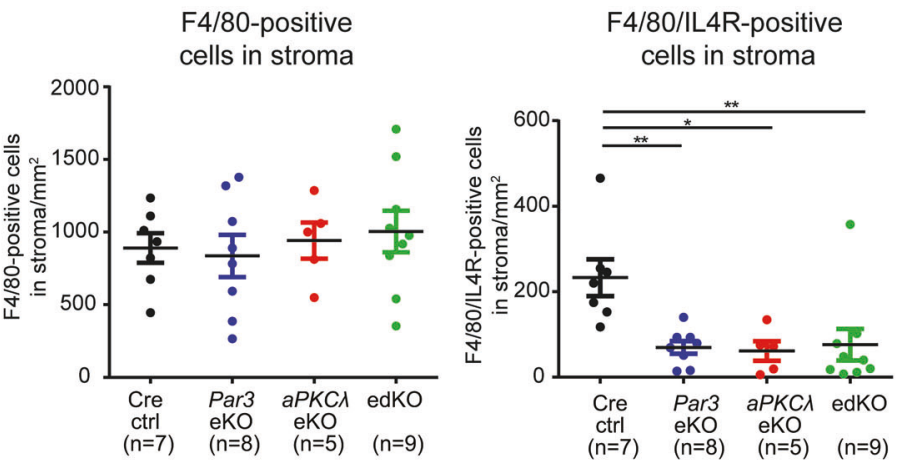

j
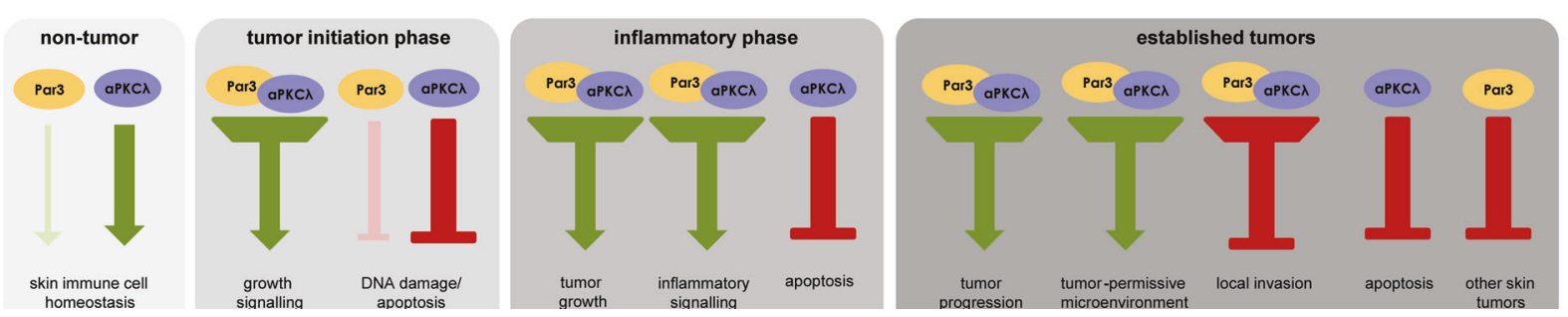

proliferation in control mice as measured by BrdU incorporation. This induction was significantly reduced in either Par3eKO or $a P K C \lambda \mathrm{eKO}$ mice, with no further decrease in edKO mice (Fig. 4a), in agreement with the lowered ERK activity induced by DMBA (Fig. $2 \mathrm{~g}$, h). Moreover, even though upon long-term DMBA/TPA treatment ERK, Akt and NF- $\mathrm{KB}$ signaling were similar (Suppl.Fig.2G,H,J,K, Suppl.Fig.3E), proliferation remained significantly reduced in all Par3/aPKC $\lambda$-mutant mice (Fig. 4a, b), albeit that single Par $3 \mathrm{eKO}$ and $a P K C \lambda \mathrm{eKO}$ mice showed a significant 
Fig. 4 Par3 and aPKC $\lambda$ control DMBA/TPA-induced early hyperproliferation and subsequent macrophage phenotypes in established tumors. a Quantification of BrdU-positive cell nuclei upon short- and long-term DMBA/TPA treatment and in untreated mice at P58 (\% of DAPI positive cell nuclei; fold change to untreated Cre $\operatorname{ctrl}=1$; mean + SEM; $n>3 /$ genotype; $* * * * p<0.0001 ; * * p<0.01 ; * p<0.05$; twoway ANOVA/Tukey's post-hoc test). b Immunofluorescence analysis as described in Fig. 2a for BrdU (\#347580, BD Bioscience) on paraffin sections of mice after long-term DMBA/TPA treatment (scale bar $=$ $100 \mu \mathrm{m})$. Nuclei were counterstained with DAPI. c Quantification of cleaved Caspase 3-positive cell nuclei upon short- and long-term DMBA/ TPA treatment and in untreated mice at P58 (\% of DAPIpositive cell nuclei; fold change to untreated Cre $\operatorname{ctrl}=1$; mean + SEM; $n>3 /$ genotype; $* * p<0.01 ; * p<0.05$; two-way ANOVA/Dunnett's post-hoc test). d Immunofluorescence analysis as previously described (2A) for BrdU (\#347580, BD Bioscience) on paraffin sections of papilloma of mice after long-term DMBA/TPA treatment (scale bar $=50 \mu \mathrm{m}$ ). Nuclei were counterstained with DAPI. e Quantification of BrdU-positive cell nuclei in papilloma after long-term DMBA/TPA treatment (BrdU-positive cells/ $\mathrm{mm}^{2}$ tumor; mean \pm SEM, one-way ANOVA/Dunnett's post-hoc test). f Quantification of cleaved Caspase 3 (cl.Casp3)-positive cell nuclei in papilloma after long-term DMBA/TPA treatment (cl. Casp3-positive cells $/ \mathrm{mm}^{2}$ tumor; mean $\pm \mathrm{SEM} ; * * p<0.01$, one-way ANOVA/Dunnett's post-hoc test). g Quantification of p53-positive cell nuclei in papilloma after longterm DMBA/TPA treatment (\% of DAPI-positive cell nuclei; $n=4$ mice/genotype; mean $\pm \mathrm{SEM}$; $* * p<0.01$; one-way ANOVA/Dunnett's post hoc test). $\mathbf{h}$ Quantification of F4/80-positive cells per $\mathrm{mm}^{2}$ in stroma of papillomas of long-term DMBA/TPA-treated mice (mean \pm SEM, one-way ANOVA/Tukey's post-hoc test). i Quantification of F4/80/IL-4R $\alpha$-double-positive cells per $\mathrm{mm}^{2}$ in stroma of papillomas of long-term DMBA/TPA-treated mice (mean \pm SEM, $* * p<0.01, * p$ $<0.05$, one-way ANOVA/Tukey's post-hoc test). j Overview scheme summarizing the findings of this study. See Suppl. Table 1 for antibody dilutions

stronger reduction than edKO mice. In conclusion, $\mathrm{aPKC} \lambda$ Par3 cooperate to induce early and sustain long-term hyperproliferation in chemical skin carcinogenesis.

We next examined how loss of Par3 or aPKC $\lambda$ affects apoptotic responses in early vs. late tumor promotion stages. Short-term DMBA/TPA treatment did not significantly alter apoptosis in Par3eKO mice, whereas apoptosis was increased in aPKC $\lambda e K O$ and edKO mice (Fig. 4c), similar to DMBA treatment alone (Fig. 2c, d), and to $a P K C \lambda$-KO keratinocytes transfected with oncogenic HRasV12 and exposed to TPA (Suppl.Videos 1, 2; Suppl. Fig. 4B). Long-term DMBA/TPA treatment also elicited an increase in cleaved Caspase3-positive cells in aPKC $\lambda \mathrm{eKO}$ and edKO mice, whilst a trend to increased apoptosis was observed in Par3eKO mice (Fig. 4c), pointing again to a differential requirement and/or timing of aPKC $\lambda$ and Par3 in counteracting cell death at different stages of tumor formation and progression.

\section{Reduced tumor growth correlates with increased apoptosis and macrophage subtype}

Par3eKO, aPKC $\lambda \mathrm{eKO}$, and edKO mice displayed significantly decreased tumor sizes compared to controls (Fig. 1b). We thus asked whether this reduction is due to reduced tumor cell proliferation. Unlike earlier tumorigenesis stages, however, BrdU incorporation, ERK, Akt, and NF-kB activities were largely comparable between tumor tissues of all experimental groups (Fig. 4d, e, Suppl.Fig. 4D-I), suggesting that tumors-once established - can bypass aPKC $\lambda$ / Par3-dependent growth signals, possibly through accumulating additional mutations [22, 23]. In contrast, apoptotic cell numbers were significantly increased particularly in aPKC $\lambda$-deficient papillomas (Fig. 4f). In agreement, only loss of aPKC $\lambda$ but not Par3 increased the number of p53positive tumor cells (Fig. 4g), potentially explaining a more pronounced reduction in tumor size in $a P K C \lambda \mathrm{eKO}$ as compared to Par3eKO mice (Fig. 1b). Together, the results identify aPKC $\lambda$ as major regulator of cell survival during DMBA-induced skin carcinogenesis. Of note, although Par3 or aPKC mislocalization has been associated with altered function in different cancers [10-14], and in skin tumors Par3 loss led to cytoplasmic redistribution of aPKC [13], aPKC $\lambda$ did not seem to control Par3 recruitment (Suppl.Fig. 4A, C).

Finally, we asked whether Par3 and/or aPKC $\lambda$ controls distinct tumor macrophage subsets that either promote or suppress tumorigenesis [37]. Whereas no change in total F4/ 80-positive (Fig. 4h) or F4/80/iNOS-double positive macrophages (not shown) in the tumor stroma was detected, the number of F4/80/IL4R $\alpha$-double-positive stromal macrophages was significantly reduced in all aPKCN/Par3-mutant mice compared to control (Fig. 4i), suggesting that epidermal aPKC $\lambda$ and Par3 next to intrinsic oncogenic signaling also promote a tumor-permissive micro-environment, which may contribute to tumor size.

Collectively, this study provides in vivo evidence that Par3 and aPKC $\lambda$ not only act in concert but also serve independent functions in skin homeostasis and tumorigenesis. Our data genetically demonstrate that Par3 and aPKC $\lambda$ act in concert (Fig. 4j) to: (1) promote early and sustained tumor outgrowth but inhibit invasion; (2) activate ERK signaling early during tumorigenesis, thus providing in vivo evidence for our previous in vitro observations showing that Par3 promotes oncogenic Ras/ERK signaling through aPKC [13]; (3) enhance epidermal Akt, NF-kB, and Stat3 signaling; (4) foster a tumor-permissive micro-environment, which taken together explain how aPKC and Par3 cooperate to (5) promote non-melanoma skin tumor incidence and burden. Interestingly, despite early changes in epidermal inflammatory signals no changes were observed in immune cell recruitment, either at early or late stages of carcinogenesis, suggesting an epidermal tumor-intrinsic role for NF- $\mathrm{KB} / \mathrm{Stat} 3$, e.g., in tumor outgrowth and DNA damage responses, in addition to promoting a protumorigenic micro-environment for tumors. Importantly, our data also provide potential evidence for a Par3- 
independent role for aPKC $\lambda$ in immune cell homeostasis as well as cell survival at different stages of tumorigenesis, whereas Par3 serves a tumor-suppressive function not shared by aPKC $\lambda$ (Fig. 4j). How aPKC $\lambda$ and Par3 control these functions will be an important future goal.

Taken together, our data show that Par3 and aPKC $\lambda$ act in concert to promote different stages of skin tumor initiation and progression. Based on the many biochemical data, they likely do so as a complex through fostering pro-proliferative, and tumor-promoting pro-inflammatory signals in epithelia executed by ERK, Akt, NF-кB, and Stat3.

Acknowledgements We thank Martim Dias Gomes for mouse line maintenance, Julia Stinn, Emmi Wachsmuth, Soriba Letzian, and Annika Schmitz for technical assistance, and the UoC animal facilities and CECAD histology unit for important services. We acknowledge Walter Birchmeier and Shigeo Ohno for sharing mouse lines, and thank the Niessen, Iden, Wickström, and Bazzi laboratories for helpful discussions. This project was funded by a joint German Research Foundation (DFG) grant (NIE-1234/4-1 and ID79/1-1 to C.N. and S. I.), a DFG-SFB832 grant to CMN (A3) and a Köln Fortune grant to S. I. (\#197/2013). Work in the Iden laboratory is also supported by the DFG (ID79/2-1, CRC829/A10). Work in the Niessen laboratory is supported by the DFG (CRC829/A01 + A05, NIE-1234/6-1) and the German Cancer Society.

\section{Compliance with ethical standards}

Conflict of interest The authors declare that they have no conflict of interest.

Open Access This article is licensed under a Creative Commons Attribution 4.0 International License, which permits use, sharing, adaptation, distribution and reproduction in any medium or format, as long as you give appropriate credit to the original author(s) and the source, provide a link to the Creative Commons license, and indicate if changes were made. The images or other third party material in this article are included in the article's Creative Commons license, unless indicated otherwise in a credit line to the material. If material is not included in the article's Creative Commons license and your intended use is not permitted by statutory regulation or exceeds the permitted use, you will need to obtain permission directly from the copyright holder. To view a copy of this license, visit http://creativecommons. org/licenses/by/4.0/.

\section{References}

1. Campanale JP, Sun TY, Montell DJ. Development and dynamics of cell polarity at a glance. J Cell Sci. 2017;130:1201-7.

2. Hirose T, Izumi Y, Nagashima Y, Tamai-Nagai Y, Kurihara H, Sakai T, et al. Involvement of ASIP/PAR-3 in the promotion of epithelial tight junction formation. J Cell Sci. 2002;115:2485-95. Pt 12

3. Yamanaka T, Horikoshi Y, Sugiyama Y, Ishiyama C, Suzuki A, Hirose T, et al. Mammalian Lgl forms a protein complex with PAR-6 and aPKC independently of PAR-3 to regulate epithelial cell polarity. Curr Biol. 2003;13:734-43.

4. McCaffrey LM, Montalbano J, Mihai C, Macara IG. Loss of the Par3 polarity protein promotes breast tumorigenesis and metastasis. Cancer Cell. 2012;22:601-14.
5. Guyer RA, Macara IG. Loss of the polarity protein Par3 activates Stat 3 signaling via an aPKC/NF- $\mathrm{KB} / \mathrm{IL}-6$ axis in mouse mammary cells. J Biol Chem. 2015;290:8457-68. 3(15)

6. Fang L, Wang Y, Du D, Yang G, Tak Kwok T, Kai Kong S, et al. Cell polarity protein Par3 complexes with DNA-PK via Ku70 and regulates DNA double-strand break repair. Cell Res. 2007;17:100-16.

7. Ishiuchi $\mathrm{T}$, Takeichi M. Willin and Par3 cooperatively regulate epithelial apical constriction through aPKC-mediated ROCK phosphorylation. Nat Cell Biol. 2011;13:860-6.

8. Nolan ME, Aranda V, Lee S, Lakshmi B, Allred DC, Muthuswamy SK. The polarity protein Par6 induces cell proliferation and is overexpressed in breast cancer. Cancer Res. 2008;68:8201-9.

9. Iden S, Rehder D, August B, Suzuki A, Wolburg-Buchholz K, Wolburg H, et al. A distinct PAR complex associates physically with VE-cadherin in vertebrate endothelial cells. EMBO Rep. 2006;7:1239-46.

10. Nakamura H, Nagasaka K, Kawana K, Taguchi A, Uehara Y, Yoshida M, et al. Expression of Par3 polarity protein correlates with poor prognosis in ovarian cancer. BMC Cancer. 2016;16:897.

11. Xue B, Krishnamurthy K, Allred DC, Muthuswamy SK. Loss of Par3 promotes breast cancer metastasis by compromising cell-cell cohesion. Nat Cell Biol. 2013;15:189-200.

12. Kojima Y, Akimoto K, Nagashima Y, Ishiguro H, Shirai S, Chishima $\mathrm{T}$, et al. The overexpression and altered localization of the atypical protein kinase $\mathrm{C}$ lambda/iota in breast cancer correlates with the pathologic type of these tumors. Hum Pathol. 2008;39:824-31.

13. Iden S, van Riel WE, Schäfer R, Song J-Y, Hirose T, Ohno S, et al. Tumor type-dependent function of the Par3 polarity protein in skin tumorigenesis. Cancer Cell. 2012;22:389-403.

14. Tervonen TA, Partanen JI, Saarikoski ST, Myllynen M, Marques E, Paasonen K, et al. Faulty epithelial polarity genes and cancer. Adv Cancer Res. 2011;111:97-161.

15. Godde NJ, Pearson HB, Smith LK, Humbert PO. Dissecting the role of polarity regulators in cancer through the use of mouse models. Exp Cell Res. 2014;328:249-57. 1-9

16. Niessen MT, Scott J, Zielinski JG, Vorhagen S, Sotiropoulou PA, Blanpain $\mathrm{C}$, et al. aPKC controls epidermal homeostasis and stem cell fate through regulation of division orientation. J Cell Biol. 2013;202:887-900. 1-14

17. Ali NJA, Dias Gomes M, Bauer R, Brodesser S, Niemann C, Iden $\mathrm{S}$. Essential role of polarity protein Par3 for epidermal homeostasis through regulation of barrier function, keratinocyte differentiation, and stem cell maintenance. J Invest Dermatol. 2016;136:2406-16.

18. Lechler T, Fuchs E. Desmoplakin: an unexpected regulator of microtubule organization in the epidermis. J Cell Biol. 2007;176:147-54.

19. Williams SE, Beronja S, Pasolli HA, Fuchs E. Asymmetric cell divisions promote Notch-dependent epidermal differentiation. Nature. 2011;470:353-8.

20. Quintanilla M, Brown K, Ramsden M, Balmain A. Carcinogenspecific mutation and amplification of Ha-ras during mouse skin carcinogenesis. Nature. 1986;6074:78-80.

21. Abel EL, Angel JM, Kiguchi K, DiGiovanni J. Multi-stage chemical carcinogenesis in mouse skin: fundamentals and applications. Nat Protoc. 2009;4:1350-62.

22. Nassar D, Latil M, Boeckx B, Lambrechts D, Blanpain C. Genomic landscape of carcinogen-induced and genetically induced mouse skin squamous cell carcinoma. Nat Med. 2015;21:946-54.

23. McCreery M, Halliwill KD, Del RosarioR, Quigley DA, Adams DJ, Balmain A. Evolution of metastasis revealed by mutational 
landscapes of chemically induced skin cancers. Nat Med. 2015;21:1514-20.

24. Kern F, Niault T, Baccarini M. Ras and Raf pathways in epidermis development and carcinogenesis. Br J Cancer. 2011;104:229-34.

25. Segrelles C, Lu J, Hammann B, Santos M, Moral M, Cascallana JL, et al. Deregulated activity of Akt in epithelial basal cells induces spontaneous tumors and heightened sensitivity to skin carcinogenesis. Cancer Res. 2007;67:10879-88.

26. Zhang Q, Lenardo MJ, Baltimore D. 30 years of NF-kB: a blossoming of relevance to human pathobiology. Cell. 2017;168:37-57.

27. Yu H, Pardoll D, Jove R. STATs in cancer inflammation and immunity: a leading role for STAT3. Nat Rev Cancer. 2009;9:798-809.

28. Kim C, Pasparakis M. Epidermal p65/NF- $\kappa \mathrm{B}$ signalling is essential for skin carcinogenesis. EMBO Mol Med. 2014;6:970-83.

29. Tran CS, Eran Y, Ruch TR, Bryant DM, Datta A, Brakeman P, et al. Host cell polarity proteins participate in innate immunity to Pseudomonas aeruginosa infection. Cell Host Microbe. 2014;15:636-43.

30. Forteza R, Figueroa Y, Mashukova A, Dulam V, Salas PJ. Conditional knockout of polarity complex (atypical) PKC reveals an anti-inflammatory function mediated by NF-kB. Mol Biol Cell. 2016;27:2186-97.

31. Geng H, Wittwer T, Dittrich-Breiholz O, Kracht M, Schmitz ML. Phosphorylation of NF-kappaB p65 at Ser468 controls its COMMD1-dependent ubiquitination and target gene-specific proteasomal elimination. EMBO Rep. 2009;10:381-6.

32. Grivennikov S, Karin M. Dangerous liaisons: STAT3 and NF-кB collaboration and crosstalk in cancer. Cytokine Growth Factor Rev. 2010;21:11-9.
33. Chan KS, Sano S, Kataoka K, Abel E, Carbajal S, Beltran L, et al. Forced expression of a constitutively active form of Stat 3 in mouse epidermis enhances malignant progression of skin tumors induced by two-stage carcinogenesis. Oncogene. 2008;27:1087-94.

34. Chan KS, Sano S, Kiguchi K, Anders J, Komazawa N, Takeda J, et al. Disruption of Stat 3 reveals a critical role in both the initiation and the promotion stages of epithelial carcinogenesis. J Clin Invest. 2004;114:720-8.

35. Ishiguro $H$, Akimoto $K$, Nagashima $Y$, Kojima $Y$, Sasaki $T$, Ishiguro-Imagawa $\mathrm{Y}$, et al. aPKClambda/iota promotes growth of prostate cancer cells in an autocrine manner through transcriptional activation of interleukin-6. Proc Natl Acad Sci USA. 2009;106:16369-74.

36. Bonastre E, Verdura S, Zondervan I, Facchinetti F, Lantuejoul S, Chiara MD, et al. PARD3 inactivation in lung squamous cell carcinomas impairs STAT3 and promotes malignant invasion. Cancer Res. 2015;75:1287-97.

37. Sica A, Mantovani A. Macrophage plasticity and polarization: in vivo veritas. J Clin Invest. 2012;122:787-95.

38. Huelsken J, Vogel R, Erdmann B, Cotsarelis G, Birchmeier W. Beta-Catenin controls hair follicle morphogenesis and stem cell differentiation in the skin. Cell. 2001;105:533-45.

39. Hirose T, Karasawa M, Sugitani Y, Fujisawa M, Akimoto K, Ohno S, et al. PAR3 is essential for cyst-mediated epicardial development by establishing apical cortical domains. Development. 2006;133:1389-98.

40. Bandyopadhyay G, Standaert ML, Sajan MP, Kanoh Y, Miura A, Braun U, et al. Protein kinase C-lambda knockout in embryonic stem cells and adipocytes impairs insulin-stimulated glucose transport. Mol Endocrinol. 2004;18:373-83. 